\title{
Cytogenetic Mechanism of Cytoplasmic Incompatibility in the Uzifly, Exorista sorbillans (Diptera: Tachinidae)
}

\author{
Bandekodigenahalli Marappa Prakash and Hosagavi Puttegowda Puttaraju*
}

Laboratory of Seribiotechnology, Department of Sericulture, Bangalore University, Bangalore-560 056, India

Received July 21, 2006; accepted September 12, 2006

\begin{abstract}
Summary Cytogenetic mechanism of Wolbachia-induced cytoplasmic incompatibility is investigated in the uzifly, Exorista sorbillans, a parasitoid of silkworm, Bombyx mori. The results revealed the condensation of chromatin to form the paternal pronucleus upon the sperm entering in to the egg, and subsequently condensed paternal and maternal pronuclei fused to form the diploid zygote in compatible crosses. In fertilized eggs from incompatible crosses, only the maternal pronucleus had individual chromosomes. The uncondensed paternal pronucleus scattered near maternal pronucleus, and in some eggs reappear as a diffused tangled chromatin mass and tend to get fragmented during the first mitotic division. Moreover, the abnormalities in the first mitotic division extend with the fragmentation and nonorientation of chromosomes on equatorial plane of first metaphase plate. The initiation of chromosome separation in late metaphase was an indication of beginning of anaphase in compatible crosses. In case of incompatible crosses, the chromosome separation had not occurred even at late metaphase/early anaphase leading the failure of development of viable embryo. However, abnormal behavior of paternal chromatin does not interfere with mitotic division of maternal chromosomes. The mechanism is discussed with applied strategy for the management of uzifly, Exorista sorbillans.
\end{abstract}

Key words Wolbachia, Uzifly, Cytoplasmic incompatibility, Chromosomes, Fertilization, PCR.

Cytoplasmic incompatibility induced by the maternally inherited alpha-proteobacteria, Wolbachia is widespread in arthropods (Werren et al. 1995, West et al. 1998, Werren and Windsor 2000, Jeyaprakash and Hoy 2000, Kikuchi and Fukatsu 2003). In its simplest form, cytoplasmic incompatibility (CI) can be described as an embryonic lethality that occurs when Wolbachia infected males inseminated with uninfected females, as a result uninfected females have fewer descendent on an average than infected ones (Yen and Barr 1971, Hoffmann et al. 1986, Presgraves 2000, Charlat et al. 2001, Puttaraju and Prakash 2005b, c). While other 3 type of crosses are fully compatible termed as unidirectional cytoplasmic incompatibility (where by infected males mate with uninfected female, the subsequent eggs fail to produce viable offspring termed as unidirectional incompatibility) (Breeuwer and Werren 1990). Whereas bi-directional CI (where the males and females harbour different strains or multiple strains of Wolbachia than the cross is said to be bidirectionally incompatible) typically occurs in mating between infected individuals harboring different strains or multiple strains of Wolbachia (O'Neill and Karr 1990). The means by which Wolbachia induced CI are currently unknown. However, there is a general prediction that Wolbachia must some how modify the paternal chromatin (Callaini et al. 1997) and this modified paternal chromatin is rescued in eggs, if infected with same strain of Wolbachia or combined with uninfected eggs results in the subsequent aborted karyogamy (Callaini et al. 1994). Several well-established cytological studies on the early events after fertilization revealed a sequence of anomalous events during early embryonic developments in Drosophila, Culex pipiens and Nasonia (Ryan and Sahu 1968, Callaini et al. 1994,

* Corresponding author, e-mail: puttarajuhp@hotmail.com 
Reed and Werren 1995, Lassy and Karr 1996). The paternal chromosome destruction in incompatible crosses is consistent with both the aberrant development and eventual death in diploids and production of all males in haplodiploids (Breeuwre 1997). So far, scientists have hypothesised that the mod and recs factors secreted in their male and female host respectively are specific for specific bacterial strains (Bourtzis et al. 1998, Mercot and Poinsot 1998), which induce different levels of cytoplasmic incompatibility (Giordano et al. 1995). Early research focused on characterization of $\mathrm{CI}$ and CI based control strategies with conventional sterile insect technique programmes (Laven 1967). These earlier works involved in the mass production and release of incompatible male insects to control wild populations of disease vectors such as mosquito, Culex pipiens. Recently, this CI based mechanism is of increasing interest for controlling medically and economically important insects (Zabolou et al. 2004) due to environmental and public health concerns associated with insecticide use and problems related to insecticide resistance. Keeping this in view, we have investigated the cytological mechanism of cytoplasmic incompatibility in the uzifly, E. sorbillans that harbors Wolbachia (Madhu and Puttaraju 2001, Puttaraju and Madhu 2002, Puttaraju and Prakash $2005 \mathrm{a}, \mathrm{b})$. The results are presented and discussed in this paper with a view to throw light on the Wolbachia-uzifly interaction and the prospects for using this association to suppress the uzifly, a serious parasitoid of silkworm.

\section{Materials and methods}

\section{Stock maintenance and crossing experiments}

The infected and uninfected populations of uzifly, E. sorbillans were maintained in the laboratory by feeding $8 \%$ glucose as described by Puttaraju and Prakash (2005c). Newly emerged male and female from infected and uninfected fly stocks were separated immediately to avoid mating within populations. Second day after emergence about 30 male flies were crossed with 30 female flies for about $24 \mathrm{~h}$ in both compatible (infected males $\times$ infected females) and incompatible (infected males $\times$ uninfected females) crosses for oviposition and subsequent egg collection for cytological analysis. The flies were provided with $8 \%$ glucose as food in a $12: 12 \mathrm{~h}$ light: dark condition at $25 \pm 1{ }^{\circ} \mathrm{C}$ with $65-70 \%$ relative humidity.

\section{Collection of eggs}

Three day after emergence and $24 \mathrm{~h}$ after mating, about 30 silkworm larvae were placed into the respective cages (uziflies prefer to lay eggs on silkworm but not on any other substratum) to get oviposited by the uzifly on the silkworm body for about $1 \mathrm{~min}$. Immediately, the eggs were scraped out carefully from the silkworm body surface and arranged on a glass slide for easy dissection.

\section{Chromosome preparation and photo-microscopy}

Two to $15 \mathrm{~min}$ old eggs were used for chromosome preparation with an interval of $1 \mathrm{~min}$. The eggs of each minute were dissected on a clean glass slide under stereo-dissection microscope. More than 500 eggs were used from each cross. The chorion was punctured and pulled away with a sharp micro needle, leaving the cytoplasm and yolk for proper spreading in fixative-I (acetic acid: methanol: distilled water, $1: 1: 2$ ) for about $2 \mathrm{~min}$. After $2 \mathrm{~min}$ a drop of fixative-II (acetic acid: methanol, $1: 1$ ) was added onto the smear and allowed for about $1 \mathrm{~min}$ followed by a drop of 100\% glacial acetic acid for proper spreading and fixation (Manjunatha 1993). The slides were dried in a hot plate with a surface temperature of $45^{\circ} \mathrm{C}$ and stained with $4 \%$ Giemsa for about $20 \mathrm{~min}$. The slides were washed in distilled water and visualized and photomicrograph were taken under 'LEITZ' research microscope supplemented with 'WILD' camera regulated by MPS-45 photoautomate with NOVA black and white film. The films were processed and photographs were prepared in dark room. 


\section{Genomic DNA isolation from uziflies}

DNA from individual infected and uninfected uziflies was extracted following usual stepwise methods of extraction with phenol, phenol: chloroform: isoamylalcohol, chloroform and finally precipitated with 2 volumes of double distilled alcohol in the presence of $3 \mathrm{M}$ sodium acetate (Sambrook et al. 1989). The precipitated DNA was washed in 70\% alcohol, dried and dissolved in $2 \mathrm{ml}$ of TE (20 mM Tris-HCl, $10 \mathrm{mM}$ disodium-ethylenediaminetetra-acetic acid) buffer. The DNA was subjected to RNase-A treatments followed by further reextraction with above steps, and finally dissolved in TE buffer. The concentration of $10-12 \mathrm{ng} / \mu \mathrm{l}$ was confirmed through quantification on $0.8 \%$ agarose gel using standard concentration of lambda DNA in TBE (89.2 mM Tris-HCl, $88.9 \mathrm{mM}$ Boric acid and $2.0 \mathrm{mM}$ disodium-ethylenediaminetetra-acetic acid) buffer for subsequent PCR analysis.

\section{PCR-analysis}

A polymerase chain reaction assay based on specific amplification of the 16S rRNA gene primer pair 76-99F 5'-TTG TAG CCT GCT ATC GTA TAA CT-3' and 1012-994R 5'-GAA TAG GTA TGA TTT TCA TGT-3' which were synthesised (Bangalore Genei, India) based on published sequence information for the 16S rRNA of Wolbachia pipientis (O'Neill et al. 1992) was used to detect Wolbachia in individual flies and it was carried out with PTC 200 of MJ Research Thermalcycler, in $20 \mu \mathrm{l}$ reaction mixture containing $1 \times \mathrm{PCR}$ buffer $(75 \mathrm{mM}$ Tris- $\mathrm{HCl}, \mathrm{pH} 8.8 ; 20 \mathrm{mM}$ $\left(\mathrm{NH}_{4}\right)_{2} \mathrm{SO}_{4} ; 0.01 \%$ Tween 20 and $2 \mathrm{mM} \mathrm{MgCl}$ ), $200 \mu \mathrm{M}$ dNTP's, $2.5 \mathrm{mM} \mathrm{MgCl}$, and $0.5 \mathrm{U}$ Taq DNA Polymerase (MBI-Fermentas Life Sciences, Vilnius, Lithuania, USA), 83.33 pmol of each forward and reverse primers (Bangalore Genei, Bangalore), $30 \mathrm{ng}$ template DNA and final volume of millipore water to $20 \mu \mathrm{l}$. The amplification was carried out with a cyclic condition of initial denaturation step at $94^{\circ} \mathrm{C}$ for 2 min followed by 40 cycles with denaturation step at $94^{\circ} \mathrm{C}$ for $1 \mathrm{~min}$, primer annealing at $55^{\circ} \mathrm{C}$ for $2 \mathrm{~min}$ and primer extension with the presence of Taq-DNA polymerase at $72^{\circ} \mathrm{C}$ for $2 \mathrm{~min}$ in each cycle and final extension at $72^{\circ} \mathrm{C}$ for $5 \mathrm{~min}$. The amplified PCR products were fractionated through $1.3 \%$ agarose gel electrophoresis run in $1 \times$ Tris-borate-EDTA $(89.2 \mathrm{mM}$ Tris- $\mathrm{HCl}, 88.9 \mathrm{mM}$ Boric acid and $2.0 \mathrm{mM}$ disodium-ethylenediaminetetra-acetic acid) buffer for a length of 5-6 cm at a constant volt of $65 \mathrm{~V}$ for Pharmacia power supply EPS $200 \mathrm{~V} / 400 \mathrm{~mA}$. The gel was stained with $0.4 \mu \mathrm{g} / \mathrm{ml}$ gel of ethidium bromide just prior to casting. The documentation was done with gel documentation system with Genesnap software. Triplicate PCR experiments were carried out on different occasions for reproducibility of expected band size.

\section{Results}

The PCR result confirms the presence and absence of Wolbachia in laboratory populations of infected and cured uziflies respectively (Fig. 1).

In compatible (infected male $\times$ infected female) and incompatible (infected male $\times$ uninfected female) crosses, the oocyte at the time of oviposition was at the first maturation metaphase. The subsequent stages were followed only after the entry of sperm into the oocytes (Manjunatha 1993). The first maturation division resulted in the formation of 2 daughter nuclei, which underwent second maturation division forming 4 daughter nuclei, which were clearly visible at telophase in both the cases. After second maturation division, 3 of the 4 daughter nuclei were arranged in a separate cluster representing 3 polar bodies and the remaining 1, which is located a little away from these 3 bodies, represented the egg pronucleus (Fig. 2a). The egg-pronucleus moved towards the anterior of the egg cell assuming a spherical shape. The polar bodies on the other hand migrated towards periphery and degenerated. The spherical female pronucleus, after $5 \mathrm{~min}$ of oviposition became distinct in the yolk mass (Fig. $2 \mathrm{~b}$ thick arrow). The sperm in the mean time underwent maturation to form male pronucleus after degeneration of its tail (Fig. $2 b$ thin arrow). The head portion assumed a 


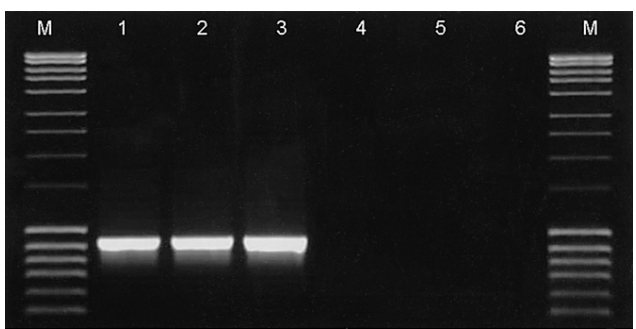

Fig. 1. PCR product of infected and uninfected (cured) uzifly populations of Exorista sorbillans. Lane M-Mass ruler (MBI-Fermentas); Lane 1, 2 and 3infected uzifly population; Lane 4, 5 and 6-uninfected uzifly population.
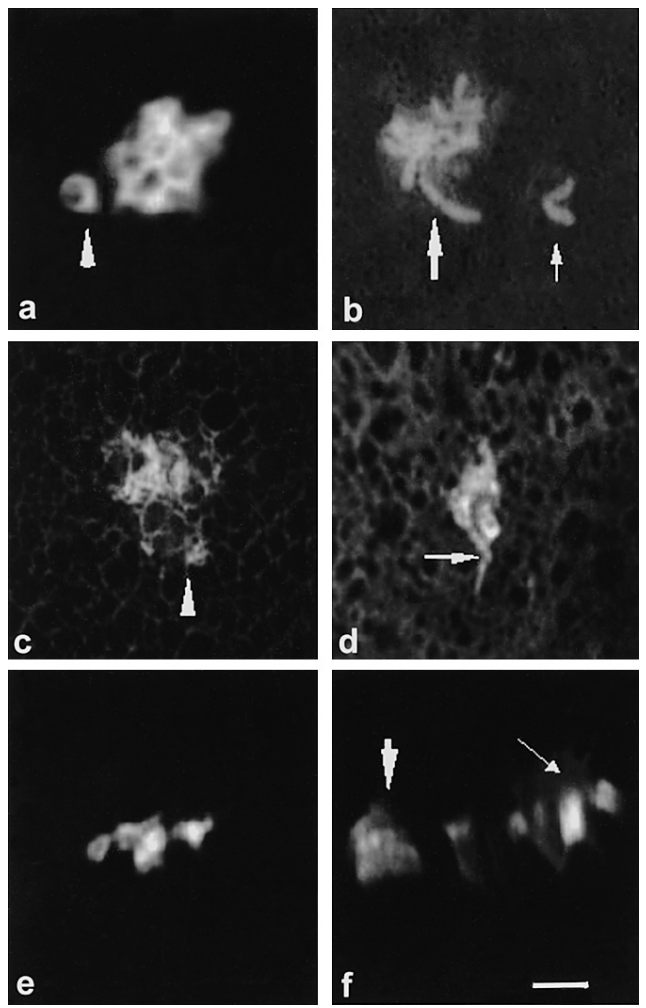

Fig. 3. Chromosome behavior of post fertilization in uzifly, Exorista sorbillans of incompatible cross (infected male $\times$ uninfected female): a. Karyogamy with aneuploidy; $b$ and $c$. Abnormal first cleavage metaphase; $d$ and e. Chromosome fail to separate from metaphase to undergo anaphase; $f$. Fragmented paternal chromatids with normal maternal chromosome. Scale bar, $\times 2000$.
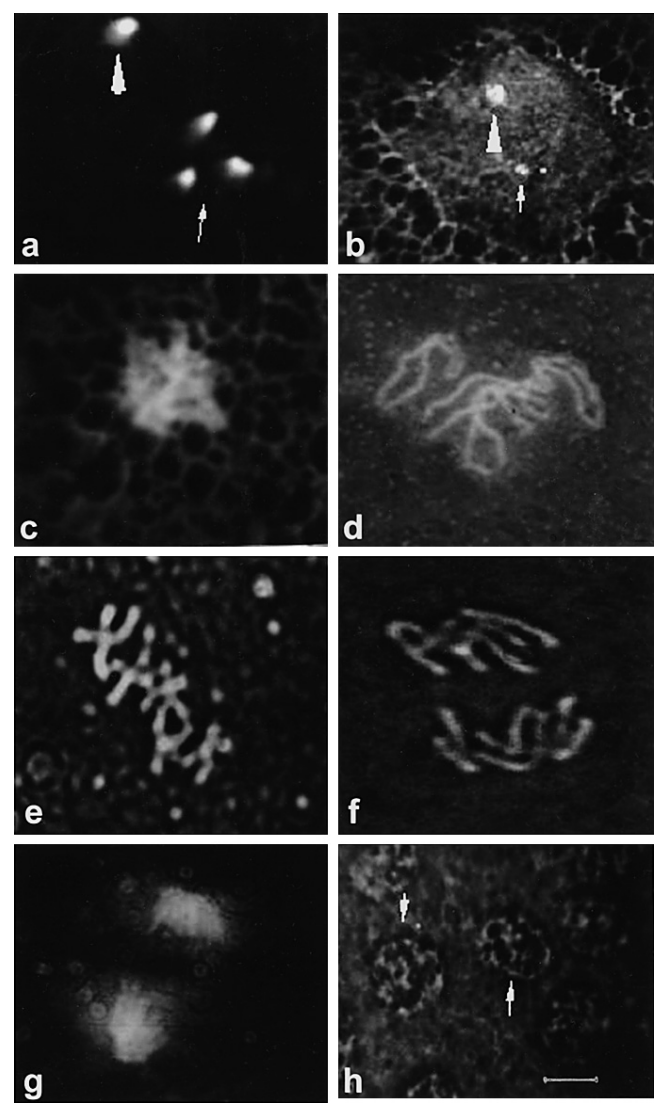

Fig. 2. Chromosome behavior of pre and post fertilization in uzifly, Exorista sorbillans of compatible cross (infected male $\times$ infected female): a. An egg pronucleus (thick arrow) and 3 polar bodies (thin arrow); b. An egg (thick arrow) and a sperm (thin arrow) pronucleus; c. Fusion of male and female pronucleus (Karyogamy); d. The first mitotic prophase; e. The first mitotic metaphase; f. The first mitotic anaphase; g. The first mitotic telophase; h. Energids (arrow). Scale bar, $\times 2000$.

spherical shape, migrated towards the female pronucleus and fused to form the synkaryon for about $8 \mathrm{~min}$ (Fig. 2c) in compatible crosses, whereas in some of incompatible crosses, fragmentation of paternal chromosomes was observed, while in some other cases incomplete karyogamy due to aneuploidy [Fig. 3a (arrow); 3b (thin arrow); 3c (arrow)]. In compatible crosses, after about 10 min of oviposition, initi-

ation of prophase was indicated (Fig. 2d). During early prophase, the chromosome condensation began resulting in the appearance of long and wavy chromosomes (Fig. 2d). The metaphase began when the chromosomes became sufficiently shorter and thicker and appeared on a equatorial plane (Fig. 2e). In case of incompatible crosses some of the chromosomes fail to arrange on equatorial plane and stay away (Fig. $3 \mathrm{~b}$ thin arrow and $3 \mathrm{c}$ thick arrowhead). The initiation of chromosome sep- 
aration in late metaphase was the indication of commencement of anaphase and all the daughter chromosomes moved towards their respective pole synchronously in late anaphase of compatible crosses (Fig. 2f). In incompatible crosses, failure of separation of chromatids from metaphase plate was observed [Fig. 3d (arrow) and 3e]. The daughter chromosomes, which moved towards opposite pole in late anaphase, lost their distinct nature and appeared as a mass in compatible crosses (Fig. $2 \mathrm{f}, \mathrm{g}$ ) while in incompatible crosses, the subsequent stages after metaphase were aborted due to fragmentation of chromatids [Fig. 3f (thick and thin arrow)]. The reconstruction began with the onset of late anaphase (Fig. $2 \mathrm{~g}$ ). The resultant daughter nuclei transformed into spherical shape followed by subsequent cleavage nuclei [Fig. 2h (arrow shows energids)].

\section{Discussion}

Although, precise mechanism of cytoplasmic incompatibility is still unclear, several excellent cytological investigations in the early stages of embryo revealed a sequence of anomalous events in insects, viz., Culex pipens (Jost 1970), Drosophila simulans (Kose and Karr 1995, Lassy and Karr 1996) and Nasonia vitripennis (Reed and Werren 1995). Number of reports suggests that, the mod and resc factors are specific for specific bacterial groups (Bourtzis et al. 1998, Mercot and Poinsot 1998), which induce abnormalities in different phases of first cleavage division of incompatible crosses that predicts the observed result. However, loss of paternal chromosome does not interfere with the sequential division of maternal chromosomes, indicating that the effect is only on paternal chromosomes. Moreover, the microtubules which were associated with first embryonic division might have affected greatly. In agreeing with a similar findings of loss of paternal set of chromosome in incompatible crosses in Nasonia vitripennis (Ryan and Saul 1968), Culex pipiens (Jost 1970) and Drosophila simulans (O'Neill and Karr 1990) it is suggested that in the cytoplasmic incompatibility bacteria prevent syngamy in incompatible crosses. In Drosophila simulans, karyological studies of cytoplasmic incompatibility indicate that a high embryonic mortality was a consequence of defects associated with first cleavage metaphase, which occur as early as in the first cleavage division (O'Neill and Karr 1990). In the wasp Nasonia, disruption of first mitosis, where impairing paternal condensation in crosses of infected male with uninfected female was reported (Ryan and Saul 1968, Breeuwer and Werren 1990, Reed and Werren 1995). Calline et al. (1997) have pointed that in incompatible crosses of Drosophila simulans, the condensation of paternal chromosomal complement was asynchronous, for because the timing of paternal chromatin condensation appeared to be delayed in these embryos. In haplodiploids, such as Nasonia sp. incompatible embryos are developed into males (Ryan and Saul 1968, Reed and Werren 1995). It was further predicted that the aneuploid nuclei were generated during karyogamy of incompatible crosses which might have developed as viable embryos. However in the later stages of maggots and pupae they die, as evidenced by the death of maggot and pupae in incompatible crosses (Prakash 2006).

Even before the causative agent of CI was known, experiments were already done to use CI caused by Wolbachia bacteria as a method for mosquito control (Laven 1967). In uzifly, expression of unidirectional cytoplasmic incompatibility in crosses between infected male and uninfected/cured females and genomic incompatibility in crosses between cured individuals (Puttaraju and Prakash 2005a-c) are suggested for the management of uzifly, a severe pest of silkworm, Bombyx mori.

\section{Acknowledgements}

Authors are thankful to DBT, New Delhi, India for financial assistance. 


\section{References}

Bourtzis, K., Dobson, S. L., Braig, H. R. and O’Neill, S. L. 1998. Rescuing Wolbachia have been overlooked. Nature 391: $852-853$

Breeuwer, J. A. J. 1997. Wolbachia and cytoplasmic incompatibility in the spinder mites Tetranychus urticae and T. turkestani. Heredity 79: 41-47.

- and Werren, J. H. 1990. Microorganisms associated with chromosome destruction and reproductive isolation between two insect species. Nature 346: 558-560.

Callaini, G., Riparbelli, M. G. and Dallai, R. 1994. The distribution of cytoplasmic bacteria in the early Drosophila, embryo is mediated by astral microtubules. J. Cell Sci. 107: 673-682.

—, Dallai, R. and Riparbelli, M. G. 1997. Wolbachia-induced delay of paternal chromatin condensation does not prevent maternal chromosomes from entering anaphase in incompatible crosses of Drosophila simulans. J. Cell Sci. 110: 271-280.

Charlat, S., Bourtzis, K. and Mercot, H. 2001. Wolbachia induced cytoplasmic incompatibility. In: Sybiosis. Seckbach, J. (ed.). Kluwer Academic Publisher, Netherland, pp. 621-644.

Giordano, R., O'Neill, S. L. and Robertson, H. M. 1995. Wolbachia infection and the expression of cytoplasmic incompatibility in Drosophila sechellia and Drosophila mauritiana. Genetics 140: 1307-1317.

Hoffmann, A. A., Turelli, M. and Simmons, G. M. 1986. Unidirectional incompatibility between populations of Drosophila simulans. Evolution 40: 692-701.

Jeyaprakash, A. and Hoy, M. A. 2000. Long PCR improves Wolbachia DNA amplification: WSP sequence found in $76 \%$ of sixty-three arthropod species. Insect Mol. Biol. 9: 393-405.

Jost, E. 1970. Untersuchungen Zur Inkompatibilität in Culex-pipiens-komplex. Wilhelm Roux’ Arch. 166: 173-188.

Kikuchi, Y. and Fukatsu, T. 2003. Diversity of Wolbachia endosymbionts in heteropterans bugs. Appl. Envior. Microbiol. 69: 6082-6091.

Kose, H. and Karr, T. L. 1995. Organization of Wolbachia pipientis in the Drosophila fertilized egg and embryo revealed by anti-Wolbachia monoclonal antibody. Mech. Dev. 51: 275-288.

Lassy, C. W. and Karr, T. L. 1996. Cytological analysis of fertilization and early embryonic development in incompatible crosses of Drosophila simulans. Mech. Dev. 57: 47-58.

Laven, H. 1967. Eradication of Culex pipiens fatigans through cytoplasmic incompatibility. Nature 261: $383-384$.

Madhu, M. and Puttaraju, H. P. 2001. Cytological and molecular evidence for Wolbachia infection in uzi flies of Exorista species. Cytologia 66: 197-203.

Manjunatha, H. B. 1993. Morphological and Cytological investigations on the uzifly, Exorista sorbillans. Ph.D. Thesis, Bangalore University, Bangalore, India.

Merçot, H. and Poinsot, D. 1998. Rescuing Wolbachia have been overlooked and discovered as mount Kilimanjaro. Nature 391: 853 .

O’Neill, S. L. and Karr, T. L. 1990. Bidirectional incompatibility between conspecific populations of Drosophila simulans. Nature 348: 178-180.

-, Giordano, R., Colbert, A. M. E., Karr, T. L. and Robertson, H. M. 1992. 16S rRNA phylogenetic analysis of the bacterial endosymbionts associated with cytoplasmic incompatibility in insects. Proc. Natl. Acad. Sci. USA 89: 2699-2702.

Prakash, B. M. 2006. Molecular evidence for Wolbachia in sericultural insect pests and its role in controlling uzifly, Exorista sorbillans. Ph.D. Thesis, Bangalore University, Bangalore, India.

Presgraves, D. C. 2000. A genetic test of the mechanism of Wolbachia-induced cytoplasmic incompatibility in Drosophila. Genetics 154: 771-776.

Puttaraju, H. P. and Madhu, M. 2002. Presence of Wolbachia endosymbionts in different silkworm races/species and in their uzi fly parasites. J. Invertebr. Pathol. 79: 120-122.

— and Prakash, B. M. 2005a. Effects of Wolbachia in the silkworm parasitoid Exorista sorbillans (Diptera: Tachinidae). J. Insect Sci. 5: 30 .

— and - 2005b. Effects of Wolbachia-Targeted tetracycline on Host-parasitoid-symbiont interaction. Eur. J. Entomol. 102: 669-674.

— and - 2005c. Wolbachia and reproductive conflicts in the uzifly, Exorista sorbillans (Diptera: Tachinidae). Arch. Insect Biochem. Physiol. (USA) 60: 230-235.

Reed, K. M. and Werren, J. H. 1995. Induction of paternal genome loss by the paternalsex-ratio chromosome and cytoplasmic incompatibility bacteria (Wolbachia): a comparative study of early embryonic events. Mol. Reprod. Dev. 40: $408-418$.

Ryan, S. L. and Saul, G. B. II. 1968. Post-fertilization effect of incompatibility factors in Mormoniella. Mol. Gen. Genet. 103: 29-36.

Sambrook, J., Fritsch, E. F. and Miniatis, T. 1989. Molecular cloning: A Laboratory manual, 2nd edition. Cold Spring Har- 
bor Laboratory Press. Cold Spring Harbor, New York, 999 pp.

Werren, J. H., Zhang, W. and Guo, L. R. 1995. Evolution and phylogeny of Wolbachia: reproductive parasites of arthropods. Proc. Roy. Soc. London Ser. B 261: 55-63.

— and Windsor, D. M. 2000. Wolbachia infection frequency in insects: evidence for a global equilibrium? Proc. Roy. Soc. Lond. B 267: 1277-1285.

West, S. A., Cook, J. M., Werren, J. H. and Godfray, H. C. 1998. Wolbachia in two insect host-parasitoid communities. Mol. Ecol. 7: 1457-1465.

Yen, J. H. and Barr, A. R. 1971. New hypothesis of the cause of cytoplasmic incompatibility in Culex pipiens. Nature 232: $657-658$.

Zabalou, S., Riegler, M., Theodorakopoulou, M., Stauffer, C., Savakis, C. and Bourtzis, K. 2004. Wolbachia-induced cytoplasmic incompatibility as a means for insect pest population control. Proc. Natl. Acad. Sci. USA 101: $15042-15045$. 